\title{
DEVELOPMENT OF THE LATERAL MOTOR COLUMN IN THE LIMBLESS MUTANT CHICK EMBRYO'
}

\author{
MARK E. LANSER AND JOHN F. FALLON ${ }^{2}$
}

Department of Anatomy, University of Wisconsin, Madison, Wisconsin 53706

Received October 10, 1983; Revised January 30, 1984; Accepted January 30, 1984

\begin{abstract}
This is a report on the development of the lateral motor column (LMC) in the limbless mutant chick embryo. The limbless mutant was used to study the effects of the absence of a periphery on the developing nervous system. The limbless mutant provides a unique opportunity to compare the effects on the LMC of deletion of a limb caused by the genotype with those seen following surgical removal of the limb primordium. Cell counts of the total number of motoneurons in the LMC at both the brachial and lumbar levels were done in a large series of limbless embryos and on their normal siblings. In normal embryos, a substantial loss of LMC motoneurons was observed during the course of normal development. At the brachial level, $54 \%$ of the initial LMC cell population was lost between day 6 and day 18 . At the lumbar level, $40 \%$ of the initial population was lost between the 6th and 12th days of development with no further loss through day 18. An even more massive cell loss was observed in the limbless mutant LMC at both brachial and lumbar spinal cord levels between day 5 and day 12 . This resulted in the elimination of at least $85 \%$ of the motoneurons that were initially present in the limbless LMC. Our data demonstrate that the effects of peripheral deprivation on LMC development in the limbless mutant are similar to those seen following surgical removal of the periphery. The initial production of motoneurons and assembly of the LMC did not appear to be significantly affected by the mutation, while the subsequent degeneration of LMC motoneurons is greatly accelerated and increased in comparison to the normal.
\end{abstract}

A large number of the motoneurons produced in the limbinnervating segments of the chick embryo spinal cord die during the course of normal development. Prior to obvious degeneration there are no known morphological or biochemical differences between motoneurons that are destined to die and those that will survive (Chu-Wang and Oppenheim, 1978a; Oppenheim et al., 1978). Prior to the period of naturally occurring cell death nearly all of the motoneurons in the lateral motor column (LMC) sprout axons that pass out of the spinal cord via the ventral root and project to muscles in the limb that are appropriate for their position within the LMC (Chu-Wang and Oppenheim, 1978b; Oppenheim et al., 1978; Lance-Jones and Landmesser, 1981; Oppenheim, 1981a; Hollyday, 1983). Because of this phenomenon, theories that have been used to explain the occurrence of cell death in the LMC have focused on the relationship between the developing motor nerves and the developing limb musculature. Most prominent among the

\footnotetext{
${ }^{1}$ This work was supported by National Science Foundation Grant PCM-8205368. A preliminary report of this work was presented at the Third International Conference on Limb Morphogenesis and Regeneration. We would like to thank Jill L. Carrington, Allen W. Clark, Leah Dvorak, Theodore Goodfriend, John K. Harting, David B. Slautterback, and William L. Todt for their helpful criticism in the preparation of this manuscript. We also thank Ms. Lucy Taylor for preparation of the graph, Ms. Sue Leonard for typing the manuscript, and Ms. B. Kay Simandl for technical help in all parts of this work.

${ }^{2}$ To whom correspondence should be addressed.
}

current theories is the "competition hypothesis" which postulates that certain motoneurons will die as a result of their inability to compete successfully with other motoneurons for a limited number of synaptic sites on the surface of the developing musculature or for a limited amount of some "trophic substance" supplied by the developing muscles (for reviews, see Oppenheim, 1981b; Cunningham, 1982).

The chick lumbar LMC has been investigated most extensively. At this level assembly of the LMC is completed by day 6. Between days 6 and $10,40 \%$ of the motoneurons are lost. After this time no further attrition of motoneurons is observed through 5 days posthatching (Hamburger, 1975; Oppenheim et al., 1978). At the brachial level the degeneration of LMC motoneurons is both more protracted and more extensive. Between days 6 and 10,30\% of the motoneurons in the LMC undergo degeneration. In the next 11 days an additional $30 \%$ of the motoneurons are lost, so that by the time the embryo hatches only $40 \%$ of the initial population of LMC motoneurons remain (Oppenheim and Majors-Willard, 1978).

Complete removal of the periphery results in the death of nearly all of the motoneurons in the LMC. Surgical extirpation of the presumptive leg bud tissue in the chick during the 2nd day of development has little, if any, effect on the early stages of formation of the LMC. On day 6 of incubation the LMC on the experimental side contains nearly a normal complement of motoneurons when compared to the contralateral, control side. However, over the next 2 to 3 days a massive degeneration of motoneurons occurs. By day 9 only a small remnant of the LMC remains on the operated side, consisting of $10 \%$ of the 
original population of motoneurons (Hamburger, 1958; Oppenheim et al., 1978). The final number of motoneurons in the LMC is roughly proportional to the amount of limb musculature that survives the extirpation (Hamburger, 1934). Extirpation of a limb bud increases the number of motoneurons that degenerate in the LMC, but the period over which this degeneration occurs is approximately the same as that seen in normal embryos, occurring between 6 and 9 days of development (Hamburger, 1975). This observation, plus the fact that no morphological or biochemical differences have been noted between the control and the experimental side prior to the period of cell death (Oppenheim et al., 1978), has led to the suggestion that the mechanisms regulating cell death following surgical removal of the periphery might possibly be the same as those regulating naturally occurring cell death (Hamburger, 1975).

Our current investigation involves a re-examination of the effects of the lack of a periphery on the development of the LMC in the chick embryo. The object of our study is the limbless chicken first described by Prahlad et al. (1979). This mutant provides an excellent model for studying the effects of absence of the periphery on the developing LMC because it does not involve any experimental manipulation of the embryo prior to the time when it is sacrificed. Therefore, the outgrowing axons will encounter peripheral tissues that have not been altered by surgery. Briefly, the limbless mutation is an autosomal recessive mutation with $100 \%$ penetrance, such that homozygous limbless chicks develop with no limbs at all four sites but with limb girdles present. The limb buds actually develop normally until Hamburger and Hamilton's (1951) stage 18, at which point the apical ectodermal ridge fails to form and the limb bud mesoderm begins to die. By stage 22 massive necrosis is evident throughout the limb bud mesoderm. At stage 23 the entire limb bud is necrotic; few living cells are evident. By stage 24 there is no evidence of an appendage at any of the limb sites (J. F. Fallon, J. M. Frederick and B. K. Simandl, submitted for publication). We present data concerning the development of the LMC in both homozygous limbless embryos and their normal siblings at both the brachial and lumbar spinal cord levels. In addition, observations concerning the trajectory of the spinal nerves in the limbless mutant will be presented.

\section{Materials and Methods}

Embryos were obtained from the flock of heterozygous limbless carriers maintained at the University of Wisconsin, Madison. All embryos were incubated in a forced-draft incubator at $37^{\circ} \mathrm{C}$ and were left untouched until they were sacrificed. Embryos were sacrificed at 4 , $4.5,5,5.5,6,7,8,9,10,11,12$, and 18 days of incubation and that time were assayed for their phenotype by simply noting the presence or absence of limbs. Those embryos in which no limbs were present represented the homozygous limbless embryos and were grouped together and designated "limbless" in the subsequent analysis. Those embryos where the presence of normal limbs was noted were designated "normal" and were used as the control embryos in the subsequent analysis. It is stressed that control embryos consisted of both homozygous normal embryos and heterozygous limbless carriers obtained from the same flock of chickens as the homozygous limbless embryos. The majority of the 4- through 12-day embryos were fixed whole overnight in $70 \%$ ethanol, $40 \%$ formaldehyde, and glacial acetic acid in a ratio of 17:2:1 and were then stained in hematoxylin en bloc according to the method of Wenger (1951). In some of the 12-day and all of the 18-day embryos, the spine was dissected free after fixation, decalcified, and then prepared in the same manner as were the whole embryos. A few embryos at each of the early stages were silver stained en bloc according to the method of Levi-Montalcini (1949). All embryos were embedded in paraffin, serially sectioned, and mounted on slides for viewing with the light microscope. Sections were cut at $8 \mu \mathrm{m}$ for 4 through 9-day embryos and at $12 \mu \mathrm{m}$ for the older embryos.

Staging. Embryos younger than 9 days of incubation were staged according to the Hamburger (1948) spinal cord stage series. To do this, camera lucida drawings of the cross-sectioned spinal cord were made at approximately the level of the middle of the 15th spinal segment of the brachial spinal cord. At the lumbar level drawings were made at approximately the level of the middle of the 26 th segment. This was done for all 4.5- through 9-day embryos that were used for cell counts. The criteria of Hamburger (1948) were then used to assign a spinal cord stage to each of the embryos. Hamburger and Hamilton's (1951) stage numbers were also assigned to these embryos according to the synchronization of the two-stage series in Hamburger (1958). Therefore, our data for the 4.5- through 9-day embryos will be presented according to both the spinal cord stage and the Hamburger and Hamilton (1951) stage in addition to the days of incubation. Data for the 10- through 18-day embryos will be presented according to the days of incubation and will be correlated with the Hamburger and Hamilton (1951) stages.

Cell counts. Motoneuron cell counts were done on a large series of embryos ranging from 4.5 to 18 days of incubation. Only those cells that were within the LMC as identified according to the criteria of Hamburger (1975) were counted. Motoneurons were not only identified by their position within the LMC, but also by their histological appearance. They were characterize $d$ by their large size, dark stain, and the presence of one or more dense nucleoli. Only those motoneurons with at least one visible nucleolus were counted. In 4.5- to 5.5-day embryos every sixth section was counted at $\times 1000$ magnification. In 6- to 8-day embryos every eighth section was counted at $\times 1000$. In 9 to 12-day embryos every tenth section was counted at $\times 400$, and in 18 -day embryos every tenth section was counted at $\times 250$. All cell counts were done directly in the light microscope using a 100 -square ocular grid. In most embryos the left or right side was selected at random, and only one side was then counted. In some embryos both sides were counted, and no significant difference was observed between the two sides (M. E. Lanser and J. F. Fallon, unpublished observations). In the cases where both sides were counted, one side was selected at random for use in the subsequent analysis.

The mean number of motoneurons per section was calculated by dividing the total number of cells counted by the number of sections that were counted. The mean number of motoneurons per section was then multiplied by the total number of sections included in the length of the LMC to yield the total number of motoneurons in the LMC. No correction was made to account for nuclear splitting since the section's thickness was always larger than the nucleolar diameter (Konigsmark, 1970; also see Chu-Wang and Oppenheim, 1978a, b). Nevertheless, because no correction was made for the double counling of nuclei, our absolute cell counts might actually be slight overestimates of the number of LMC motoneurons; however, because we are comparing embryos that have been identically prepared, consistent increases in the absolute numbers should have little effect on the relative results (see also Hollyday and Hamburger, 1976).

Statistical comparison of limbless embryos with their normal siblings at each stage was done using the Student's two-tailed $t$ test. Degenerating cells were identified by the presence of dense, pyknotic nuclear debris. Although no precise counts of degenerating cells were done, their presence and extent were noted on each section that was counted. Also, the distribution of the spinal nerves was noted in both the hematoxylin- and the silver-stained material.

\section{Results}

Lateral motor column cell counts are listed in Table I, and the data for the brachial level are illustrated in Figure 1.

Normal embryos. Cell counts in the normal embryos were in general agreement with previously published accounts of LMC development for normal chick embryos (see Hamburger, 1975; Oppenheim and Majors-Willard, 1978). The LMC attained its maximum complement of motoneurons between the 5 th and 6 th days of development at both the brachial and lumbar levels. Subsequent degeneration of the brachial LMC resulted in the loss of $27 \%$ of the motoneurons between days 6 and 10 , followed by the loss of approximately another $27 \%$ of the initial cell population in the next 8 days. By day $18,54 \%$ of the initial population of LMC motoneurons had been lost. At the lumbar level only about $40 \%$ of the initial population of motoneurons was lost during development. The period of cell loss was not as long as at the brachial level, being completed by the 12 th day of development when approximately $60 \%$ of the initial population of motoneurons remained. 
TABLE I

Motoneuron cell counts of both brachial and lumbar lateral motor columns in both wild-type and limbless embryos

\begin{tabular}{|c|c|c|c|c|c|c|c|}
\hline & $\begin{array}{c}\text { Day } 4.5 \\
\text { Stage } 24^{a} \\
(4 \mathrm{n} 10)\end{array}$ & $\begin{array}{c}\text { Day } 5.0 \text { to } 6.0 \\
\text { Stage } 25 \text { to } \\
29 \\
(5 \mathrm{n} 2 \text { to } 5 \mathrm{n} 11)\end{array}$ & $\begin{array}{c}\text { Day } 6.5 \text { to } 7.5 \\
\text { Stage } 30 \text { to } \\
33 \\
\text { (7n1 to } 7 \mathrm{n} 10) \\
\end{array}$ & $\begin{array}{c}\text { Day } 8.0 \text { to } 8.5 \\
\text { Stage } 34 \text { to } \\
35 \\
\text { (8n11 to } 8 \mathrm{dn}) \\
\end{array}$ & $\begin{array}{c}\text { Day } 10 \\
\text { Stage } 36\end{array}$ & $\begin{array}{c}\text { Day } 12 \\
\text { Stage } 38\end{array}$ & $\begin{array}{c}\text { Day } 18 \\
\text { Stage } 44\end{array}$ \\
\hline \multicolumn{8}{|l|}{ Brachial } \\
\hline \multirow[t]{9}{*}{ Wild-type } & 13,318 & 15,056 & 11,952 & 13,231 & 8,842 & 9,697 & 7,004 \\
\hline & 14,016 & 13,557 & 13,548 & 15,217 & 11,365 & 8,107 & 6,618 \\
\hline & 12,945 & 14,523 & 14,806 & 12,069 & 12,439 & 8,265 & \\
\hline & 13,767 & 17,096 & 12,174 & 14,999 & & 11,222 & \\
\hline & 12,087 & 15,485 & 15,136 & 12,276 & & & \\
\hline & & 15,987 & 12,375 & & & & \\
\hline & & 13,812 & & & & & \\
\hline & & 14,179 & & & & & \\
\hline & & 14,390 & & & & & \\
\hline Mean & 13,227 & 14,898 & 13,331 & 13,558 & 10,882 & 9,322 & 6,811 \\
\hline Percentage of survival & & & 89.5 & 91.0 & 73.0 & 62.6 & 45.7 \\
\hline \multirow[t]{13}{*}{ Limbless } & 11,289 & 13,526 & 6,006 & 3,657 & & 1,931 & 1,540 \\
\hline & 13,351 & 11,621 & 5,211 & 3,753 & & 1,770 & 1,856 \\
\hline & 12,553 & 11,462 & 5,102 & 3,493 & & 1,980 & \\
\hline & 9,567 & 10,192 & 5,009 & 3,658 & & & \\
\hline & & 11,184 & & 3,558 & & & \\
\hline & & 15,085 & & & & & \\
\hline & & 10,021 & & & & & \\
\hline & & 9,776 & & & & & \\
\hline & & 11,857 & & & & & \\
\hline & & 8,785 & & & & & \\
\hline & & 10,074 & & & & & \\
\hline & & 8,557 & & & & & \\
\hline & & 9,496 & & & & & \\
\hline Mean & 11,690 & 10,895 & 5,332 & 3,624 & & 1,894 & 1,698 \\
\hline Percentage of survival & & 93.2 & 45.6 & 31.0 & & 16.2 & 14.5 \\
\hline \multicolumn{8}{|l|}{ Lumbar } \\
\hline \multirow[t]{10}{*}{ Wild-type } & & 16,915 & & & & 9,431 & 11,052 \\
\hline & & 17,173 & & & & 10,541 & 11,078 \\
\hline & & 17,976 & & & & 9,657 & \\
\hline & & 19,354 & & & & 12,108 & \\
\hline & & 16,475 & & & & & \\
\hline & & 18,908 & & & & & \\
\hline & & 19,288 & & & & & \\
\hline & & 19,971 & & & & & \\
\hline & & 18,145 & & & & & \\
\hline & & 18,816 & & & & & \\
\hline Mean & & 18,302 & & & & 10,434 & 11,064 \\
\hline Percentage of survival & & & & & & 57.0 & 60.4 \\
\hline \multirow[t]{8}{*}{ Limbless } & 13,952 & 17,448 & & & & 1,822 & 1,896 \\
\hline & 14,687 & 14,216 & & & & 2,557 & 1,903 \\
\hline & & 13,626 & & & & 2,690 & \\
\hline & & 13,788 & & & & 2,388 & \\
\hline & & 10,995 & & & & & \\
\hline & & 16,487 & & & & & \\
\hline & & 14,106 & & & & & \\
\hline & & 16,909 & & & & & \\
\hline Mean & 14,319 & 14,696 & & & & 2,364 & 1,899 \\
\hline Percentage of survival & & & & & & 16.0 & 12.9 \\
\hline
\end{tabular}

"The top number indicates Hamburger and Hamilton's (1951) stage; the number in parentheses indicates Hamburger's (1948) spinal cord stage.

Limbless embryos. In contrast to the normal embryos which reached a peak LMC cell population between 5 and 6 days of development, the limbless mutant LMC attained its maximum complement of motoneurons at the brachial level almost a full day earlier, at 4.5 days. While at this stage the assembly of the normal LMC has not yet been completed (Hamburger, 1975; Hollyday and Hamburger, 1977), comparable cell counts of presumptive LMC motoneurons in limbless and normal embryos demonstrated the presence of fewer motoneurons in the limbless LMC (13, 227 versus 11,190). This difference was not statistically significant ( $p>0.1$ ). However, between days 5 and 6 , when formation of the normal LMC is being completed (Hamburger, 1975; Hollyday and Hamburger, 1977), the limbless brachial LMC actually showed a $7 \%$ loss of motoneurons. Since during this same interval the normal LMC has continued to add motoneurons, the difference between the normal and limbless embryos increased to such an extent that by the 6 th day of development there were approximately 4000 fewer mo- 


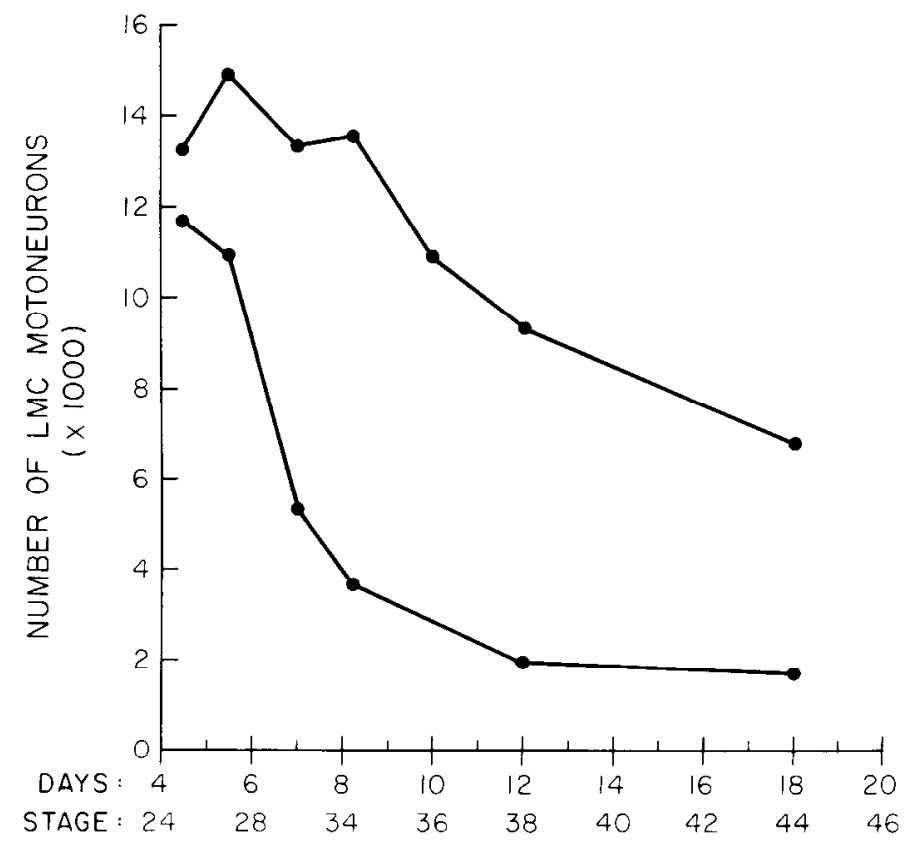

Figure 1. Development of the brachial LMC. The mean numbers of motoneurons in the brachial LMC from Table I are plotted for both normal (upper curve) and limbless mutant (lower curve) embryos as a function of the developmental age of the embryos in days of incubation. It can be seen that degeneration of the normal brachial LMC begins during the 6th day of development and continues at least through the 18th day of development. On the other hand, degeneration of the limbless brachial LMC, while beginning at approximately the same time as in the normal, is completed earlier, at least by the 12th day of development. Also notice that degeneration of the normal LMC is initially much more gradual than that seen in the limbless mutant which exhibits a very precipitous degeneration between 5.5 and 7 days of development.

toneurons in the limbless $\mathrm{LMC}$ at both the brachial and lumbar levels, a difference that was highly significant $(p<0.001)$. At all stages investigated after the 6th day of development (days 6.5 to $7.5,8.0$ to $8.5,12$, and 18 ), the limbless LMC contained significantly fewer motoneurons when compared to the normal $(p<0.001)$.

Between days 6.0 and 7.5 of development over one-half of the total number of motoneurons that were present in the limbless brachial LMC at 4.5 days of development underwent degeneration, so that by the end of the 7th day of development only about $45 \%$ of the motoneurons remained. Continued degeneration of motoneurons during the 8th day of development accounted for the loss of an additional $15 \%$ of the 4.5-day population, leaving only about $30 \%$ of the initial population of motoneurons intact by the end of day 8 . In contrast, during the 7 th and 8th days of development in normal embryos the brachial LMC exhibited only a $10 \%$ reduction in motoneuron number. In other words, while degeneration of the brachial LMC in the normal was only about $20 \%$ completed by the end of the 8th day of development, it was over $80 \%$ completed in the limbless mutant. By day 12 , when degeneration of the normal brachial LMC was only $70 \%$ completed, degeneration in the limbless LMC had ceased. Hence, at the brachial level cell death in the limbless LMC was greatly accelerated when compared to the normal. However, at the lumbar level degeneration was completed in the LMC of both the limbless and normal embryos by the 12th day of development.

In summary, at both the brachial and lumbar levels of the limbless mutant $85 \%$ of the initial population of motoneurons was lost by day 12. In normal embryos there was both a different time course and extent of LMC degeneration at the two levels.
Fifty-four percent of the initial normal population was lost by the 18th day at the brachial level, while $40 \%$ of the initial population was lost by the 12th day at the lumbar level. Figures 2 through 4 illustrate the degeneration of the brachial LMC in normal and limbless embryos.

Degenerating cells were observed in the LMC of both limbless and normal embryos at 4.5 days of development, but their occurrence was extremely rare. Beginning at 5 days of development and continuing through the 8th day, large numbers of degenerating cells were seen in the LMC of limbless embryos. While necrotic cells were also observed in normal embryos at these stages, there appeared to be fewer dead cells than were observed in the limbless embryos. While a quantitative analysis of cell death was not done as part of the present investigation, it did appear that the number of degenerating cells observed at any given stage was roughly proportional to the number of motoneurons lost from the LMC during that stage as indicated by the cell counts.

The developing spinal nerves in the limbless mutant appeared to travel along appropriate pathways as they approached the vacant limb site until they reached the shoulder girdle, at which point they seemed to end abruptly (Fig. 5). Occasionally, small nerve bundles were observed leaving the terminal portion of the spinal nerve, and sometimes even approaching the body wall, but it was impossible to ascertain whether these were composed of motor nerve axons. A "neuroma," a structure consistently found in embryos that have had a limb bud surgically extirpated (Hamburger, 1958; Oppenheim et al., 1978), was never observed in limbless embryos. During the 6th day of development a large amount of cellular debris was seen in the terminal nerve bundle and was coincident with the period of massive cell loss in the limbless LMC. This observation was in general agreement with electron microscopic evidence of phagocytic cells containing axonal debris in the area of the neuroma on day 5, following leg bud extirpation (Oppenheim et al., 1978). Finally, it was observed that the limbless embryos also possessed a grossly apparent reduction in size of the spinal ganglia and dorsal horns when compared to normal embryos at both 12 and 18 days of development.

\section{Discussion}

The observations presented in this report indicate that the failure of the periphery to develop in the limbless mutant chick embryo appears to have little, if any, effect on the initial production of motoneurons or their migration into the LMC and initial differentiation. Rather, the lack of periphery exerts its effect on the subsequent survival of these motoneurons, accelerating and increasing the amount of cell death. Our data indicate that LMC development in the limbless mutant is similar to LMC development following surgical extirpation of the limb primordium. However, there is one significant difference between the two. This difference is the observation that the spinal nerves of the limbless mutant follow a ventrolateral trajectory that is quite unlike the direct ventral projection with neuroma formation seen following extirpation (Hamburger, 1958; Oppenheim et al., 1978).

Motoneuron survival. While the absence of a periphery increases the number of cells dying at the earliest stages of LMC degeneration, it seems as though this absence is not causing cells to die any earlier in the limbless LMC than in the normal. This conclusion is supported by the observation that degenerating cells were observed in both the limbless and the normal LMC at 4.5 days (stage 24). While fewer cells are present in the limbless brachial LMC when compared to the normal LMC at 4.5 days, it cannot presently be determined whether this is due to "hypoplasia" or "hyperthanasia" of motoneurons in the limbless LMC (cf. Hamburger, 1975). The apparent loss of cells in the brachial LMC of the limbless mutant between day 4.5 

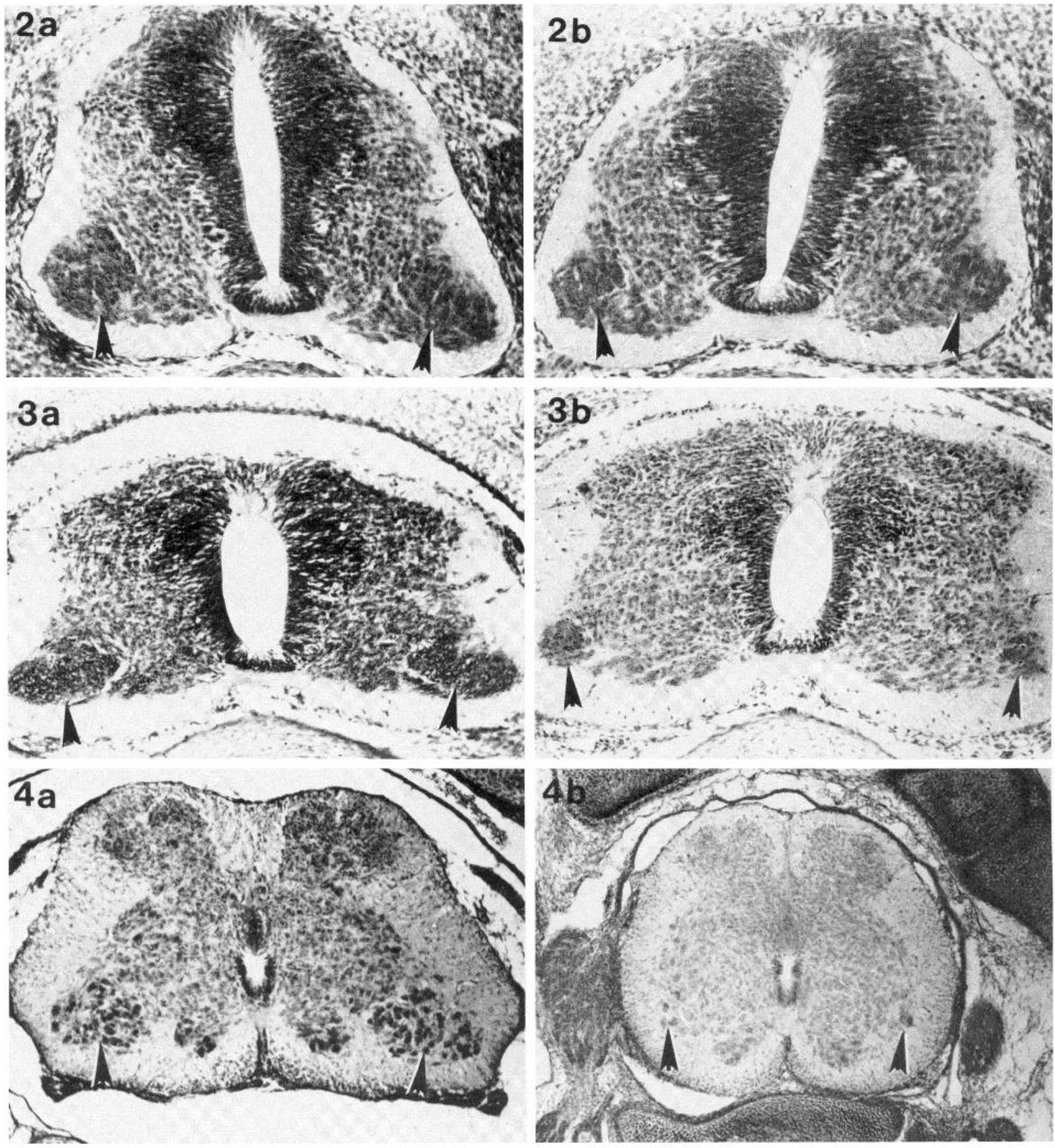

Figure 2. Cross-section through segment 15 of the brachial spinal cord in 5.5-day normal (a) and limbless (b) chick embryos. The LMC is the dark cluster of cells located in the ventrolateral portion of the spinal cord and is indicated by the arrowheads. Note the diminished size of the LMC in the limbless compared to the normal. Magnification: $a, \times 136 ; b, \times 157$.

Figure 3. Cross-section through segment 15 of the brachial spinal cord in 7.5-day normal $(a)$ and limbless $(b)$ chick embryos. The LMC is the dark cluster of cells in the ventrolateral portion of the spinal cord and is indicated by the arrowheads. Note the great disparity in size of the limbless and normal LMCs. Also, compare the limbless LMC with that at 5.5 days (Fig. $2 b$ ) and notice the reduction in size. Contrast this with the normal which shows little decrease in size between 5.5 (Fig. $2 a$ ) and 7.5 days. Magnification: $a, \times 126 ; b, \times 122$.

Figure 4. Cross-section through segment 15 of the brachial spinal cord in 12-day normal $(a)$ and limbless $(b)$ chick embryos. The LMC is the dark cluster of cells located in the ventrolateral portion of the spinal cord and is indicated by the arrowheads. Despite the difference in appearance between the two, they are from the same developmental stage and spinal cord level. Note the continued shrinkage of the LMC in the limbless mutant which now consists of a small cluster of only a few cells, and compare to the normal, which is still a large cluster of cells. Magnification: $a, \times 80 ; b, \times 59$.

and days 5 to 6 (stages 25 to 29 ) most likely can be attributed to the large number of motoneurons undergoing degeneration at these early stages of the cell death period. This is in contrast to the normal brachial LMC, where relatively few motoneurons undergo degeneration at these early stages and an increase in motoneuron number is seen because of the continued migration of motoneurons into the LMC. Clearly, the lack of a periphery exerts its effect on the survival of motoneurons. The precise mechanisms that mediate this effect await further investigation; however, given the differing temporal patterns of LMC 


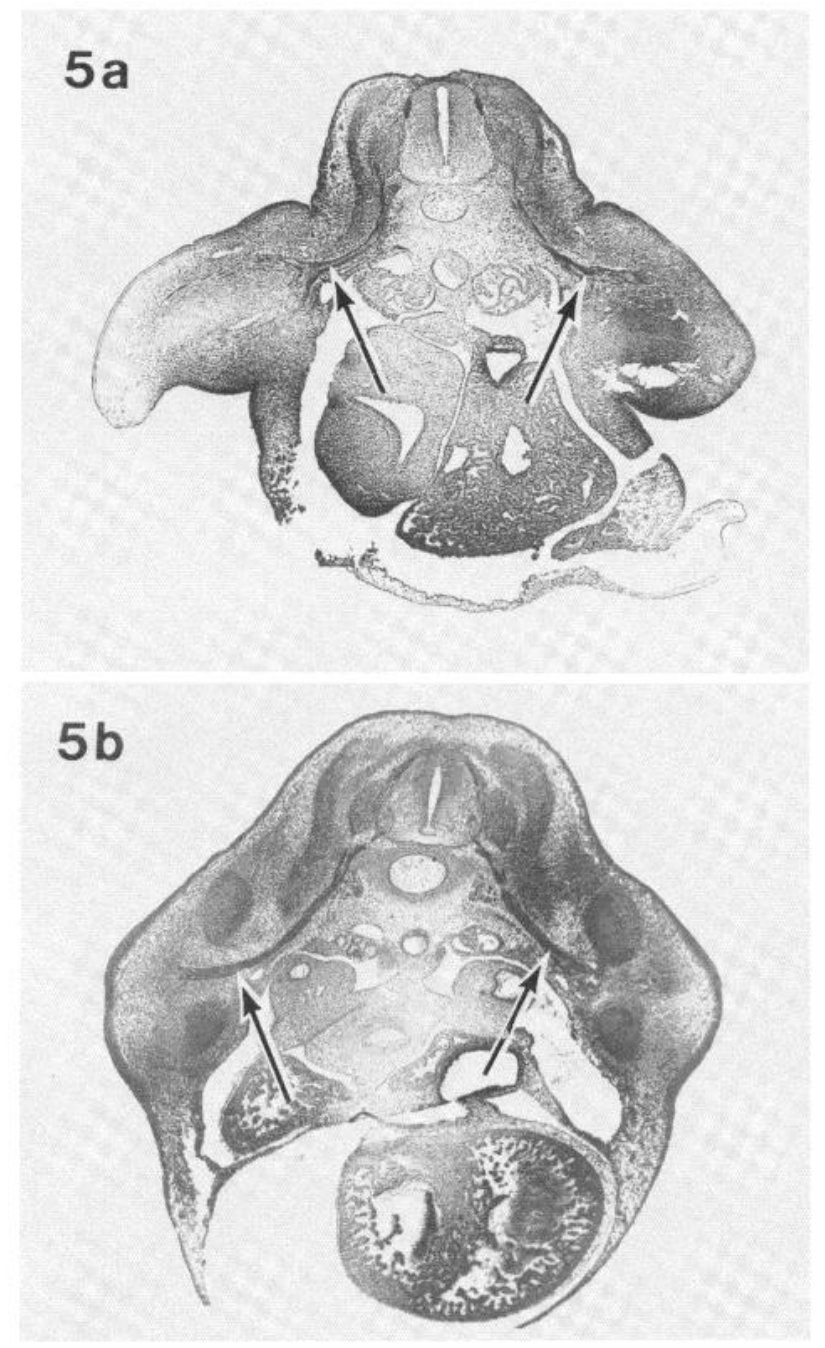

Figure 5. Pathways of the spinal nerves in a 4.5-day normal $(a)$ and a 5-day limbless $(b)$ embryo that were en bloc silver stained. Note the similarity between the trajectory of the spinal nerves as they approach the vacant limb site in the limbless mutant and in the normal. The arrows indicate the spinal nerves. There appears to be no obvious difference between the limbless mutant and the normal until the limb girdle is reached. At this point the axons in the limbless mutant appear to end in an abrupt manner, while those in the normal exhibit the characteristic dorsal and ventral branching pattern into the limb. Magnification $\times 28$.

degeneration in limbless and normal embryos at the brachial level, it is not unreasonable to speculate that different mechanisms may be responsible for the bulk of motoneuron degeneration in the two cases. It may be that primary interaction with the periphery normally stabilizes the entire population of motoneurons and is requisite for their initial survival. The failure to establish this interaction due to the absence of a periphery could then result in an accelerated cell death pattern due to the increased number of dying cells during the early stages of the cell death period.

Although cell death in limbless and normal brachial LMCs appears to begin at the same time, the subsequent course of this process is markedly different in the two. In the brachial LMC, cell death occurs over a longer time interval in normal embryos than in limbless embryos or in embryos that have had a wing bud surgically removed (Hamburger, 1958; Oppenheim and Majors-Willard, 1978). This should be compared with the lumbar LMC, where cell death is completed over approximately the same time interval in normal and limbless embryos and in embryos that have had a leg bud surgically removed (Hamburger, 1958, 1975; Oppenheim et al., 1978). It is not clear why brachial LMC motoneurons die over a shorter interval following peripheral deletion than in normal embryos. Furthermore, it is not clear why naturally occurring cell death in the brachial LMC occurs over a longer time interval than in the lumbar LMC. The reasons for the protracted time course of degeneration in the normal brachial LMC when compared to the normal lumbar LMC or the brachial and lumbar LMCs following peripheral deletion require further investigation.

In normal chick embryos the wing musculature develops from cells derived from the somites adjacent to the developing wing bud (Chevallier et al., 1977; Christ et al., 1977). The myogenic cells of the somite begin migration into the somatopleure in the region of the future wing bud at stage 13 (Jacob et al., 1978). The presumptive myoblasts are present in the wing bud but are histologically indistinguishable from other mesenchymal cells in the limb bud until stage 24 , when myoblast differentiation and segregation into dorsal and ventral muscle masses begins (Sullivan, 1962; Chevallier et al., 1977; Bennett et al., 1980, 1983; also see Bennett, 1983; Hollyday, 1983). Lateral motor column motoneurons are born from stage 17 , when the limb buds first form, through stage 24 (Hollyday and Hamburger, 1977). Between stages 18 and 22 the outgrowing axons of these motoneurons collect at the tip of the myotome. At stage 23 the axons arrive at the limb bud base, and finally at stage 24 the axons enter the limb bud (see Hollyday, 1983).

In limbless chick embryos the limb buds appear to develop normally until stage 18 , at which point the apical ectodermal ridge fails to form. Subsequently, between stages 18 and 24 , the entire limb bud mesoderm undergoes massive necrosis, such that by the end of stage 24 there is no limb bud tissue at all four sites (J. F. Fallon, J. M. Frederick, and B. K. Simandl, submitted for publication). Therefore, at the time when axons would be expected to grow into the developing limb bud (stage 24 ), there is no recognizable limb bud tissue in limbless embryos, although the limb girdle elements and some associated musculature remain. Gross dissection of 18-day limbless embryos reveals that all limb muscles derived from the dorsal and ventral muscle masses in the wing bud, including $\mathrm{M}$. pectoralis, are absent; while those girdle muscles derived from the axial system, e.g., M. rhomboideus superficialis, are intact (for normal chick developmental anatomy, see Sullivan, 1962). In addition, there are muscles associated with the pelvic girdle in the limbless mutant. Identification of the muscles in this region is complicated by the fact that all limb skeletal elements are absent, although it does appear that obturator externus and possibly gluteus superficialis and profundus are present (for normal chick anatomy, see Chamberlain, 1943). Although no studies comparable to Sullivan (1962) are available for the leg musculature, it is reasonable to speculate that these muscles are derived from axial elements of the myotome as opposed to the dorsal and ventral muscle masses of the leg bud.

Eighty-five percent of the motoneurons initially present in the LMC at both the brachial and lumbar levels die during the course of development of the limbless mutant chick embryo. This result is nearly the same as the $90 \%$ reduction in motoneuron number observed in the LMC following surgical removal of the wing (Hamburger, 1958) or the leg (Hamburger, 1958; Oppenheim et al., 1978) primordium. Previous reports (Hamburger, 1934) have indicated that the number of motoneurons remaining in the LMC following wing bud extirpation is roughly proportional to the amount of muscle tissue that survives the operation. More recent studies have indicated that the motoneurons which die following segmental deletion of hindlimb structures are those which would be expected to innervate the missing structures based on their position within the LMC (Whitelaw and Hollyday, 1983). 
Extensive degeneration of the LMC in the limbless mutant precludes direct comparison to motor pool maps of the normal chick limb innervation pattern (see Hollyday, 1980). However, it is possible to make some general conclusions based on the rostrocaudal distribution of surviving motoneurons in the limbless LMC. At both the brachial and lumbar levels it is apparent that the greatest number of surviving motoneurons are located in the more rostral segments of the LMC (M. E. Lanser and J. F. Fallon, unpublished observations). This is where one would expect to find motoneurons that innervate the more proximal muscles in the normal limb, such as those associated with the shoulder girdle (Hollyday, 1980). While not ruling out the possibility that the LMC motoneurons which survive the period of cell death following limb bud extirpation are innervating residual limb musculature, Oppenheim et al. (1978) have suggested that there may be a small number of LMC cells that may not be dependent on their innervation target for survival. More specific information regarding the peripheral innervation targets of the surviving motoneurons in the limbless mutant by utilizing both retrograde and anterograde transport of horseradish peroxidase (HRP) is now being sought.

Development of the LMC in limbless chickens resembles the transient existence of the LMC seen during development of snake-like, limbless reptiles (Anguis fragilis) described by Raynaud et al. (1977). The development of the LMC in both limbless chickens and the limbless reptiles differs from that observed in true snakes such as Tropidonotus tessellata and Python reticulatus, where a distinct LMC is never produced (Raynaud et al., 1977). The nervous system of snakes shows no capacity to innervate a limb during development. In contrast, the nervous systems of limbless chickens and of apodal reptiles do show this capacity, but it is never realized, presumably due to motoneuron degeneration resulting from a primary failure of limb development.

Spinal nerve trajectory. A consistent and possibly significant difference observed between the limbless mutant and embryos that have had their limb buds surgically removed is in the peripheral distribution of the outgrowing axons. Even in embryos where the lateral body wall has reformed following the extirpation, the outgrowing axons end in a characteristic neuroma and do not approach the vacant limb site (Oppenheim et al., 1978). In all cases that we have investigated, the outgrowing axons in limbless mutant embryos approach the vacant limb site in a ventrolateral trajectory resembling that seen in the normal control embryos (see Fig. 5).

It is obvious that the outgrowing axons in the two cases encounter radically different conditions upon leaving the spinal cord and approaching the periphery. In normal embryos, outgrowing axons gather at the ventral tip of the myotome at stage 18 and remain there until stage 23 , at which point they begin to invade the limb bud tissue (Hollyday, 1983). In embryos where the limb bud has been surgically removed prior to stage 18 , the outgrowing axons do not encounter any limb bud tissue. In the limbless mutant there is a limb bud present during the period of axon outgrowth and residence at the tip of the myotome. Although the limb bud is undergoing degeneration during stages 19 to 22 , it is, nonetheless, present and may influence the developing motor axons. The formation of a neuroma following extirpation of the limb primordium and its absence following failure of the periphery to develop in the limbless mutant may be due to the damaging effect of surgical intervention on axonal pathway signals. However, it is not possible to rule out other plausible explanations. One possibility is that the periphery, which is present for a longer time in the limbless mutant than in embryos that have had a limb bud surgically removed, may exert a tropic effect on the outgrowing axons.

Since the limbless gene affects the apical ectodermal ridge (J. F. Fallon, J. M. Frederick, and B. K. Simandl, submitted for publication) the possibility exists that the nervous system, which is derived from the ectoderm, is also affected by the gene. Considering the similarity of our results to those seen in the LMC following limb bud extirpation (Hamburger, 1958; Oppenheim et al., 1978), we feel this is unlikely. However, it is now possible to address directly whether the gene affects the nervous system because we have developed a technique to permit wing development in homozygous limbless embryos (J. L. Carrington and J. F. Fallon, submitted for publication). We plan to use this technique to investigate further the effects of the periphery on the development of the LMC in the limbless mutant.

\section{References}

Bennett, M. R. (1983) Development of neuromuscular synapses. Physiol. Rev. 63: 917-1048.

Bennett, M. R., D. F. Davey, and K. E. Uebel (1980) The growth of segmental nerves from the brachial myotomes into the proximal muscles of the chick forelimb during development. J. Comp. Neurol. 189: 335-357.

Bennett, M. R., D. F. Davey, and J. J. Marshall (1983) The growth of nerves in relation to the formation of the premuscle cell masses in the developing chick forelimb. J. Comp. Neurol. 215: 217-227.

Chamberlain, F. W. (1943) Atlas of Avian Anatomy-Osteology, Arthrology, Myology, Michigan Agricultural Experiment Station Memoir Bulletin 5, Hallenbeck Printing Co., East Lansing, MI.

Chevallier, A., M. Kieny, and A. Mauger (1977) Limb-somite relationship: Origin of the limb musculature. J. Embryol. Exp. Morphol. 41: 245-258.

Christ, B., H. J. Jacob, and M. Jacob (1977) Experimental analysis of the origin of the wing musculature in avain embryos. Anat. Embryol. 150: $171-186$.

Chu-Wang, I. -W., and R. W. Oppenheim (1978a) Cell death of motoneurons in the chick embryo spinal cord. I. A light and electron microscopic study of naturally occurring and induced cell loss during development. J. Comp. Neurol. 177: 33-58.

Chu-Wang, I. -W., and R. W. Oppenheim (1978b) Cell death of motoneurons in the chick embryo spinal cord. II. A quantitative and qualitative analysis of degeneration in the ventral root, including evidence for axon outgrowth and limb innervation prior to cell death. J. Comp. Neurol. 177: 59-86.

Cunningham, T. J. (1982) Naturally occurring neuron death and its regulation by developing neural pathways. Int. Rev. Cytol. 74: 163186.

Hamburger, V. (1934) The effects of wing bud extirpation on the development of the central nervous system in chick embryos. J. Exp. Zool. 68: 449-494.

Hamburger, V. (1948) The mitotic patterns in the spinal cord of the chick embryo and their relation to histogenetic processes. J. Comp. Neurol. 88: 221-283.

Hamburger, V. (1958) Regression versus peripheral control of differentiation in motor hypoplasia. Am. J. Anat. 102: 365-409.

Hamburger, V. (1975) Cell death in the development of the lateral motor column of the chick embryo. J. Comp. Neurol. 160:535-546.

IIamburger, V., and H. L. Hamilton (1951) A series of normal stages in the development of the chick embryo. J. Morphol. 88: 49-92.

Hollyday, M. (1980) Organization of motor pools in the chick lumbar lateral motor column. J. Comp. Neurol. 194: 143-170.

Hollyday, M. (1983) Development of motor innervation of chick limbs. In Limb Development and Regeneration, Proceedings of the Third International Conference on Limb Morphogenesis and Regeneration, Part A, J. F. Fallon and A. I. Caplan, eds., pp. 183-193, Alan R. Liss, New York.

Hollyday, M., and V. Hamburger (1976) Reduction of the naturally occurring motor neuron loss by enlargement of the periphery. J. Comp. Neurol, 170: 311-320.

Hollyday, M., and V. Hamburger (1977) An autoradiographic study of the formation of the lateral motor column in the chick embryo. Brain Res. 132: 197-208.

Jacob, M., B. Christ, and H. J. Jacob (1978) On the migration of myogenic stem cells into the prospective wing region of chick embryos. A scanning and transmission electron microscope study. Anat. Embryol. 153: 179-193.

Konigsmark, B. W. (1970) Methods for the counting of neurons. In 
Contemporary Research Methods in Neuroanatomy, W. J. H. Nauta and S. O. E. Ebbesson, eds., pp. 315-380, Springer-Verlag, Inc., New York.

Lance Jones, C., and L. Landmesser (1980) Pathway selection by chick lumbosacral motoneurons during normal development. Proc. R. Soc. Lond. Biol. 214: 1-18.

Levi-Montalcini, R. (1949) The development of the acoustico-vestibular centers in the chick embryo in the absence of the afferent root fibers and of descending fiber tracts. J. Comp. Neurol. 91: 209-241.

Oppenheim, R. W. (1981a) Cell death of motoneurons in the chick embryo spinal cord. V. Evidence on the role of cell death and neuromuscular function in the formation of specific peripheral connections. J. Neurosci. 1: 141-151.

Oppenheim, R. W. (1981b) Neuronal cell death and some related regressive phenomena during neurogenesis: A selective historical review and progress report. In Studies in Developmental Neurobiol ogy, Essays in Honor of Viktor Hamburger, W. M. Cowan, ed., pp. 74-133, Oxford University Press, New York.

Oppenheim, R. W., and I. -W. Chu-Wang (1977) Spontaneous cell death of spinal motoneurons following peripheral innervation in the chick embryo. Brain Res. 125: 154-160.

Oppenheim, R. W., and C. Majors-Willard (1978) Neuronal cell death in the brachial spinal cord of the chick is unrelated to the loss of polyneuronal innervation in wing muscle. Brain Res. 154: 148-152.
Oppenheim, R. W., I. -W. Chu-Wang, and J. L. Maderdrut (1978) Cell death of motoneurons in the chick embryo spinal cord. III. The differentiation of motoneurons prior to their induced degeneration following limb bud removal. J. Comp. Neurol. 177: 87-112.

Pittman, R., and R. W. Oppenheim (1979) Cell death of motoneurons in the chick embryo spinal cord. IV. Evidence that a functional neuromuscular interaction is involved in the regulation of naturally occurring cell death and the stabilization of synapses. J. Comp. Neurol. 187: 425-446.

Prahlad, K. V., G. Skala, D. G. Jones, and W. E. Briles (1979) Limbless: A new genetic mutant in the chick. J. Exp. Zool. 209: 422-434.

Raynaud, A., P. Clairambault, S. Renous, and J. -P. Gasc (1977) Organisation des cornes ventrales de la moelle épinière, dans les régions brachiale et lombaire, chez les embryons de Reptiles serpentiformes et de Reptiles à membries bien développés. C. R. Acad. Sci. [D] (Paris) 285: 1507-1509.

Sullivan, G. E. (1962) Anatomy and embryology of the wing musculature of the domestic fowl (Gallus). Aust. J. 7ool. 10: 458-516.

Wenger, B. S. (1951) Determination of structural patterns in the spinal cord of the chick embryo studied by transplantations between brachial and adjacent levels. J. Exp. Zool. 116: 123164.

Whitelaw, V., and M. Hollyday (1983) Thigh and calf discrimination in the motor innervation of the chick hindlimb following deletions of limb segments. J. Neurosci. 3: 1199-1215. 